\title{
A comparison of three response models incorporated in the latent class models for the analysis of "pick any/n" data
}

\author{
HIROSHI HOJO \\ 6-3-1-58-401, Lchibancho, Tachikaze-shi, Tokyo 190
}

\begin{abstract}
This paper presents two new response models for the analysis of preference or dominance data called "pick any $/ n$ " data. The one assumes that in Stage 1 a subject determines the number of stimuli to be chosen as most preferred, and in Stage 2 he involuntarily ranks $m$ (=the number determined) stimuli out of all $n$ stimuli, giving that partial ranking as a "pick any $/ n$ " response, and the other assumes that in Stage 2 the subject finds a set of $m$ stimuli every member of which is more preferred to all the remaining $n-m$ stimuli, providing this set as a response. These response models besides the conventional threshold model are implemented in the latent class models. The analyses of nine sets of "pick any $/ n$ " data by these three variants of the latent class model indicate that the two new models better fit the data when $n$ is relatively small $(n \leqq 6)$; otherwise, the threshold model gives a better account of the data
\end{abstract}

Key words: latent class models, "pick any $/ n$ " data, preferences, response models, classification.

In the "pick any $/ n$ " method subjects are given a set of $n$ stimuli and are asked to choose in random order any number of them according to some evaluation criterion (e.g., their preferences for those stimuli). For the analysis of any $/ n$ picking data (in this paper we use the word "any/n picking" for "pick any $\left./ n^{\prime \prime}\right)$ the so-called response threshold model to be described soon is always postulated as a response model of any $/ n$ picking behavior. The response model, in general, is a psychological process model that reflects the psychological processes involved in a specific task situation which generates a specific type of data (Takane, 1981). Usually the response model postulates the rules for decisions or choices which relate the stimulus representations to the specific type of data, and provides formulas to predict the probabilities of all possible responses. Thus, in our view, this response model should be deemed a central part of the psychological scaling method.

Generally speaking, when we apply the response model to any $/ n$ picking data observed in a random sample of subjects from a particular population, we tacitly assume that all members of this population perceive and evaluate the stimuli in essentially the same way. This strong assumption of complete homogeneity in the population is certainly untenable in particular when measuring subjects' preferences. At the same time, however, if we assume that all subjects are different from each other in their perception and evaluations, the number of parameters to be estimated increases with the number of subjects, leading to difficult estimation and inferential problems.

One major approach to this problem is to assume, instead of assuming full heterogeneity among subjects, that heterogeneity is restricted and can be described by grouping subjects with small intragroup and large intergroup differences. The latent class model (Lazarsfeld \& Henry, 1968) provides a straightforward modeling framework for this approach. Böckenholt and Böckenholt's (1991) analysis is its good example. It is true that their analysis and other similar studies (e.g., Takane, 1983) are far superior to the conventional ones without taking into account the heterogeneity of subjects. If viewed, however, from the position taken here that the response model is important part of the scaling method, they are somewhat unsatisfactory in that they have not asked the question whether the response 
threshold model is only one possible response model of any $/ n$ picking behavior, ruling out other possibilities.

In this paper another two response models of any $/ n$ picking behavior are proposed, and an attempt is made to accommodate these three different response models to the case where subjects are sampled out from a nonhomogeneous population by linking them to latent class analysis so as to compare the goodness of fit of the three models using various sets of any $/ n$ picking data.

In the subsequent section those three different response models, which are designated the threshold model, the partial ranking model, and the dichotomizing model, respectively, will be provided.

\section{The Models}

\section{The Threshold Model}

Let $A=\{1, \ldots, n\}$ be a set of stimuli under study, and $a_{i}$ be the scale value of stimulus $i$. Suppose here that $a_{i}$ represents the degree of preference for stimulus $i$. We assume that when stimulus $i$ is perceived by subject $k$, the preference value $a_{i}$ is perturbed by the error process

$$
y_{i k}=a_{i}+e_{i k},
$$

where $y_{i k}$ is an error perturbed metric process for stimulus $i$ and subject $k$, and where

$$
e_{i k} \sim \mathcal{N}\left(0, s^{2}\right) .
$$

The key assumption of this model is that stimulus $i$ is picked as most preferred if and only if the error perturbed scale value $y_{i k}$ exceeds some response threshold $c$. Assume further that stimuli are judged independently of each other.

From the above assumptions it follows that the number of possible any $/ n$ picking response patterns is $2^{n}-1$ (where 1 is the case in which no stimuli are chosen). We denote the set of stimuli chosen by subject $k$ by $M(k)$, and call this set response pattern $M(k)$, or more simply response $M$ when there is no need to specify $k$. Then, the probability, $v[M(k)]$, that response $M(k)$ is internally generated is given by

$$
v[M(k)]=\left\{\prod_{i \in M_{i}(k)} F_{i}\right\}\left\{\prod_{j \in H(j)}\left(1-F_{j}\right)\right\},
$$

where $F_{i}$ is the probability that the error perturbed preference value $y_{i t}$ is above the response threshold $c$, and where

$$
H(k)=A-M(k),
$$

that is, $H(k)$ is the set of those stimuli not chosen as most preferred by subject $k$; the letter $H$ will be used when there is no need for specifying $k$. Under the normality assumption (2), $F_{i}$ is obtained by

$$
F_{i}=\operatorname{Pr}\left[y_{i k}>c\right]=\int_{b i}^{\infty} \phi(z) d z,
$$

where $\phi(z)$ is the standard normal probability density function, and where $z=\left(y_{i k}-a_{i}\right) / s$ and $b_{i}=\left(c-a_{i}\right) / s$.

Let $v[M(o)]$ be the probability that no stimuli are chosen as most preferred. This $v[M(o)]$, which is computed by (3) setting $H(k)=A$, should be excluded from the computation of the probability, $p[M(k)]$, that response $M(k)$ is actually given. Thus, we have

$$
p[M(k)]=\frac{v[M(k)]}{\sum_{l=1}^{2 \cdot-1} v[M(l)]}=\frac{v[M(k)]}{1-v[M(0)]},
$$

where $M(l)$ stands for the $l$-th response pattern of all $2^{n}-1$ different response patterns.

(It must be noted that although the response threshold model on the basis of the Luce's (1959) choice model which Takane (1983) and Böckenholt and Böckenholt (1991) used in their analyses looks different from the one given above, the two are essentially the same. See also Hojo (1986) for a more detailed description of the threshold model.)

Now, $L$, the joint likelihood for the entire set of observations is stated as

$$
L=\prod_{k=1}^{N} p[M(k)]
$$

where $\mathcal{N}$ is the number of subjects. Suppose 
that all $\mathcal{N}$ response patterns produced by subjects are divided into $R$ distinct patterns. Let us denote this set of all $\mathrm{R}$ distinct responses obtained in the experiment by $\Phi$, each of which is referred to as $M(r)(\mathrm{r}=1, \ldots$, $R$ ). Then the above (7) is also expressed as

$$
L=\prod_{r=1}^{R} p[M(r)]^{\mathcal{M}_{(r)}},
$$

where $\mathcal{N}(r)$ is the number of subjects who give response $M(r)$. The above $L$ will be used later in the estimation procedure.

\section{The Partial Ranking Model}

This response model distinguishes two stages in the preference judgment process. In Stage 1 the subject is assumed to determine how many stimuli he will choose as most preferred from the set of $n$ stimulus alternatives. This number of stimuli to be chosen is called the NSC (Number of Stimuli to be Chosen) number, letting NSC be the variable to represent the NSG number for convenience. Assume further that subjects determine their respective NSC numbers independently of each other. We use the symbol $u_{m}$ to denote the probability that the NSC number which any particular subject selects is $m(m=1, \ldots, n)$.

After the number $m$ is selected as the NSC number in Stage 1, the subject in Stage 2 is assumed to continue ranking $n$ stimuli from 1 to $m$, and to stop ranking after having chosen the $m$-th most preferred stimulus. This assumption is not so unnatural since it is probable that the subject picks out the $m$ most preferred stimuli from $n$ stimuli by involuntarily ranking them despite the fact that his task is not ranking but any $/ n$ picking. Of course, however, there is no guarantee that the subject writes $m$ stimuli down accroding to his preference orders for these $m$ stimuli. Therefore, the subject's response as it stands cannot be taken as a ranking judgment even if stimuli are actually chosen by ranking as this model assumes. Rather, it must be regarded as any one outcome of all possible rankings for $m$ stimuli in $M(k)$.

Let us refer to the partial ranking where only the $m$ most preferred stimuli of all $n$ are rank ordered (with $n-m$ stimuli left unordered) as the $m / n$ ranking (which corresponds to "rank $m / n$ " in Takane \& de Leeuw, 1987). The number of all possible ways to rank $m$ stimuli in $M(k)$ is computed by $m$ factorial, namely, $m !$. Then, $\Psi_{M(k)}$, a set of all possible $m / n$ rankings for set $M(k)$ has $\mathrm{m}$ ! elements; the $h$-th element of $\Psi_{M(k)}$ is designated by $g\left(h, \Psi_{M(k)}\right)$.

Now, the probability, $p\left[g\left(h, \Psi_{M(k)}\right)\right]$, that $g\left(h, \Psi_{M(k)}\right)$ is generated is derived as follows (Takane \& Carroll, 1981). Let $(i, k) \in g(h$, $\Psi_{M(k))}$ denote the stimulus judged to be the $i$-th most preferred by subject $k$. Then the probability, $p(i, k)$, that stimulus $(i, k)$ is chosen as the $i$-th most preferred stimulus is given by

$$
\begin{gathered}
p(i, k)=\operatorname{Pr}\left[y_{(i, k) k}>y_{(i+1, k) k, \ldots,}, y_{(i, k) k}>\right. \\
y_{\left.(m, k) k, \ldots, y_{(i, k) k}>y_{(n, k) k}\right],}
\end{gathered}
$$

which can well be approximated by the following multivariate logistic distribution when (1) and (2) are assumed:

$$
p(i, k)=\left[1+\sum_{j=i+1}^{n} \exp \left\{D\left(a_{(i, k)}-a_{(j, k)}\right)\right\}\right]^{-1},
$$

where $D$ is a dispersion parameter which is approximately $\pi /(\sqrt{6} s)$. The probability of a complete $m / n$ ranking, or $p\left[g\left(h, \Psi_{M(k)}\right)\right]$, is given by

$$
p\left[g\left(h, \Psi_{M(k)}\right)\right]=\prod_{i=1}^{m} p(i, k) .
$$

According to our assumption given above the probability that response $M(k)$ is generated after the selection of the number $m$ for the NSC number is equal to the sum of the probabilities of all $m ! m / n$ rankings for $M(k)$. Consequently, if $p[M(k) \mid \mathcal{N S C}=m]$ denotes the conditional probability that $M(k)$ occurs, assuming $\mathcal{N S C}=m$, we derive

$$
p[M(k) \mid \mathcal{N S C}=m]=\sum_{h=1}^{m !} p\left[g\left(h, \Psi_{M(k)}\right)\right] .
$$


Thus, $p[M(k)]$, the probability that subject $k$ yields response $M(k)$ is

$$
p[M(k)]=u_{m(k)} p[M(k) \mid \mathcal{N S C}=m(k)],
$$

where $m(k)$ denotes the NSG number that subject $k$ selects. The joint likelihood for the entire set of observations is the same as given in (7) or (8).

\section{The Dichotomizing Model}

The third response model is also a twostage model with the same assumption about Stage 1 as in the partial ranking model. The key idea of the present model comes from the view that an any $/ n$ picking is obtained as a result of distinguishing only the most preferred stimuli (to be in $M$ ) from the remaining ones (to be in $H$ ) in a dichotomous way. On the basis of this idea we assume that an any $/ n$ picking response is produced whenever an individual's preference value of every stimulus in $M$ is greater than his preference values of all stimuli in $H$.

This dichotomizing model is described formally as follows. First, let us define $\Omega_{m}$ as a set of all possible subsets of $A=\{1, \ldots, n\}$ each of which contains $m$ elements. The total number of these subsets is computed by ${ }_{n} C_{m}$, namely, the number of different ways to choose $m$ elements from $n$ elements, and the symbol $M\left(h, \Omega_{m}\right)$ is used to denote the $h$-th member of $\Omega_{m}$. It is necessary here to realize that one and the same set $M$ can be expressed by either $M(k)$ or $M\left(h, \Omega_{m}\right)$. Similarly, $H(k)$ is expressed by $H\left(h, \Omega_{m}\right)=A-M(h$, $\left.\Omega_{m}\right)$. Indeed, we let $M(k)=M\left(h, \Omega_{m}\right)$ in the equation as will be seen later.

Now suppose that the NSC number selected by subject $k$ in Stage 1 is $m$. In Stage 2 he chooses at random one element of $\Omega_{*}$ or $M\left(h, \Omega_{m}\right)$. It follows from the above assumption that when the subject's judgment is $\left\{y_{i k}>y_{j, k}, \ldots, y_{i k}>y_{j_{a-m}}\right\}$ for every $i \in M\left(h, \Omega_{m}\right)$, where $j_{1}, \ldots, j_{n-m}$ are the elements of $H\left(h, \Omega_{m}\right)$, he yields that set, namely, $M\left(h, \Omega_{m}\right)$, as his response; otherwise, he chooses another set of $m$ stimuli from $\Omega_{m}$. Then he perceives $y_{*}$ and $y_{j k}$ for every $i \in M$ and all $j \in H$, and judges whether $\left\{y_{i k}\right\}$ and $\left\{y_{j k}\right\}$ satisfy all requirements to elicit an any/ $n$ picking response, proceeding in this way until a set $M$ randomly chosen from $\Omega_{m}$ meets the constraints to generate a response.

Now, denote by $d\left[M\left(h, \Omega_{m}\right)\right]$ the probability that $y_{i k}$ becomes larger than $y_{1^{n}}, \ldots, y_{j_{t-m^{k}}}$ for every $i \in M\left(h, \Omega_{m}\right)$, then we have

$$
d\left[M\left(h, \Omega_{m}\right)\right]=\prod_{i=1}^{m} \operatorname{Pr}\left[y_{i k}>y_{1} k, \ldots, y_{i k}>y_{j_{n}-m^{k}}\right] .
$$

As in the computation of (9) or (10), if we assume (1) and (2), each $\operatorname{Pr}\left[y_{i k}>y_{j_{1}, k}, \ldots\right.$, $\left.y_{i k}>y_{j_{n-m} k}\right]$ on the right hand side of (14) is given by

$$
\begin{aligned}
\operatorname{Pr}\left[y_{i k}\right. & \left.>y_{j, k}, \ldots, y_{i k}>y_{j_{n-m}}\right] \\
& =\left[1+\sum_{j \in H\left(h_{i}, \Omega_{m}\right)} \exp \left\{D\left(a_{i}-a_{j}\right)\right\}\right]^{-1} .
\end{aligned}
$$

The point to be emphasized here is this: We have assumed that every element of $\Omega_{m}$ has a uniform probability to be chosen, i.e., $1 /{ }_{n} C_{m}$. This implies that the probability that set $M\left(h, \Omega_{m}\right)$ is chosen from $\Omega_{m}$ and $\left\{y_{i k}\right\}$ $(i \in M)$ and $\left\{y_{j k}\right\}(j \in H)$ satisfy the above conditions needed to provide an any/ $n$ picking response is proportional to $d\left[M\left(h, \Omega_{m}\right)\right]$. Cionsequently, if we denote by $p\left[M\left(h, \Omega_{m}\right) \mid \mathcal{N S C}\right.$ $=m]$ the probability that response $M\left(h, \Omega_{m}\right)$ occurs after the selection of the NSC number equal to $m$, we derive

$$
p\left[M\left(h, \Omega_{m}\right) \mid \mathcal{N S C}=m\right]=\frac{d\left[M\left(h, \Omega_{m}\right)\right]}{\sum_{q=1} d\left[M\left(q, \Omega_{m}\right)\right]} .
$$

Now, if we write $M\left(h(k), \Omega_{m}\right)$ to make it explicit that the any/ $n$ picking response which subject $k$ produces corresponds to the $h(k)$-th element of $\Omega_{m}$, then the probability that subject $k$ provides response $M\left(h(k), \Omega_{m}\right)$ $=M(k)$ actually occurs is computed by

$$
p[M(k)]=u_{m(k)} p\left[M\left(h(k), \Omega_{m}\right) \mid N S C=m(k)\right] .
$$

The joint likelihood for the entire set of observations is the same as given in (7) and (8). 
Latent Class Models for Any/n Picking Data

Basic to the latent class model is the assumption that the non-homogeneous population can be divided into a set of $T$ homogeneous subpopulations or latent classes, each of them characterized by a distinctive set of scale values which are assumed to govern the any $/ n$ pickings of the subject belonging to that particular class. Thus, we use the symbol $a_{u}(i=1, \ldots, n ; t=1, \ldots, T)$, in place of $a$, to represent the scale value of stimulus $i$ for class $t$.

Let $p_{t}[M(k)]$ be the probability that any $/ n$ picking response $M(k)$ is given within latent class $t$. This $p_{i}[M(k)]$ is obtained in almost the same fashion as $p[M(k)]$ except that $a_{u}$, the response threshold $c_{t}$ and the dispersion parameter $s_{i}$ for class $t$ should be used for $a_{i}, c$, and $s$, respectively. Denote by $\pi$, the probability that a randomly selected subject belongs to latent class $t$. Then we obtain the following expression for the probability $p[M(k)]$ that response $M(k)$ is observed when sampling is from the entire population:

$$
p[M(k)]=\sum_{i=1}^{T} \pi_{i} p_{i}[M(k)] .
$$

Obviously, the parameters $\pi_{\text {i }}$ satisfy the constraint

$$
\sum_{i=1}^{T} \pi_{i}=1
$$

The above argument is equally true of all three respnose models incorporated in their respective latent class models, each of which may be considered a variant of the latent class model for the analysis of any/ $n$ picking data.

\section{Parameter Estimation}

For our three variant latent class models, the parameters $\left\{a_{i t}\right\},\left\{c_{i}\right\},\left\{s_{i}\right\}$, and $\left\{u_{m}\right\}$ are to be estimated. Since the EM algorithm has been applied successfully for estimating latent class model parameters in a variety of applications (e.g., Goodman, 1979; Croon, 1989; Böckenholt \& Böckenholt, 1991), we also take this algorithm (Croon, 1989) to estimate the above parameters.

The E-step first determines the frequencies $f_{i}[M(r)]$ with which the $r$-th any $/ n$ picking response pattern $M(r)$ in $\Phi$ is observed within each latent class $t$. During this step the provisional estimates of parameters are used to determine the any $/ n$ picking probabilities $p_{t}[M(r)]$. Then the following weights $w_{t}[M(r)]$ are computed:

$$
w_{i}[M(r)]=\frac{\pi_{i} p_{i}[M(r)]}{\sum_{i=1}^{T} \pi_{i} p_{i}[M(r)]}
$$

in which the provisional estimates $\pi$ of the latent class probabilities are used. These weights represent the conditional (or posterior) probabilities that response $M(r)$ originated from latent class $t$. Finally, the unobserved frequency $f[M(r)]$ is estimated by

$$
f[M(r)]=w_{i}[M(r)] f[M(r)],
$$

where $f[M(r)]$ is the observed frequency of response $M(r)$ in the entire sample.

During the M-step of our iterative procedure, the maximum likelihood estimates of the parameters and of the latent class probabilities are determined. The estimates of the latent class probabilities are easily computed in the following way:

$$
\pi_{\mathrm{r}}=\frac{1}{\mathcal{N}} \sum_{r=1}^{R} f[M(r)]
$$

The new values of the other parameters are estimated by the quasi-Newton method in the present analysis. We used a BASIC program of the quasi-Newton method written by Tone (1981). These new values are then used during the next E-step. In this way, one can reach the maximum of the likelihood function by alternating the E- and Msteps.

Now, let $l$ be the $\log$ of $L$ in (8):

$$
l=\sum_{r=1}^{R} \mathcal{N}(r) \ln p[M(r)] .
$$

This $l$ is actually maximized with respect to 
the model parameters. A few comments may be needed for the case of two-stage models. Consider, for example, the following likelihood for the partial ranking variant of the latent class model:

$$
\begin{aligned}
l & =\sum_{r=1}^{R} \mathcal{N}(r) \ln \left[u_{m(r)} p[M(r) \mid \mathcal{N S C}=m(r)]\right] \\
= & \sum_{m=1}^{n} n(m) \ln u_{m}+\sum_{r=1}^{R} N(r) \ln p[M(r) \mid N S C \\
& =m(r)],
\end{aligned}
$$

where $n(m)$ is the observed frequency with which the number $m$ is selected as the NSC number, and $m(r)$ denotes the number of stimuli in the $r$-th response pattern $M(r)$. Letting the first term be $l$ and the second term $l_{2}, l$ is expressed as $l=l_{1}+l_{2}$. Define $\operatorname{MAX}(l)$ as the likelihood $l$ maximized over its associated parameters. Then we have $\operatorname{MAX}(l)=\operatorname{MAX}\left(l_{1}\right)+\operatorname{MAX}\left(l_{2}\right)$ because the paramters used in $l$ and $l_{2}$ are not related to each other.

Under the assumption made in the twostage model it is obvious that the observations $\{n(1), \ldots, n(n)\}$ are multinomially distributed with the parameters $\left\{u_{1}, \ldots, u_{n}\right\}$. Accordingly, as is well known, the maximum likelihood estimators of the $u_{m}$ in $l_{1}$ are $n(m) / \mathcal{N}$; therefore, $\operatorname{MAX}\left(l_{1}\right)$ becomes constant. In fact, thus, the quasi-Newton iteration is performed to maximize only $l_{2}$. The same argument is true of the dichotomizing variant of the latent class analysis.

In order to compare the goodness of fit of the models we apply the AIC statistic (Akaike, 1974). The AIC statistic is defined for model $\alpha$ as

$$
\mathrm{AIC}=-2 \mathrm{MAX}(l)+2 d(\alpha),
$$

where $d(\alpha)$ is the effective number of parameters in model $\alpha$. The numbers of free parameters in the threshold, partial ranking, and dichotomizing variants of the latent class model, which are referred to as $d(T h r$.$) ,$ $d($ Par. $)$, and $d($ Dic. $)$, respectively, are each computed by

$d(T h r)=.n \times T$ (for the scale values) $+T$ (for
$\left.s_{i}\right)+T$ (for threshold $\left.c_{t}\right)-T$ (for arbitrary unit of the scale values for each $t)-T$ (for arbitrary origin of the scale values for each $t)+(T-1)\left(\right.$ for $\left.\pi_{i}\right)$

$d($ Par. $)=n \times T$ (for the scale values) $+T$ (for s) $-T$ (for arbitrary unit of the scale values for each $t)-T$ (for arbitrary origin of the scale values for each $t)+(T-1)\left(\right.$ for $\left.\pi_{i}\right)+(n-$ 1) (for $u_{m}$ )

$$
d(\text { Dic. })=d(\text { Par. }) .
$$

\section{Examples of Application}

Nine sets of any $/ n$ picking data to be analyzed here are summarized in Table 1 . We call these nine sets of data Data $1, \ldots, 9$, respectively. The students in introductory statistics courses participated in the experiments.

These data were analyzed by the threshold, partial ranking, and dichotomizing response models within the framework of latent class model. Unfortunately, however, when $n$ was greater than 6 , the partial ranking model was not applicable since the computing time and memory space required exceeded the power of our small computer (HP-86, Hewlett Packard). (Consider, for example, $7 !=5040$ in (12).) What is worse, for Data 4, 5, 6, 7, 8, and 9, sample sizes were too small relative to the numbers of possible response patterns to estimate the parameters and to determine the true number of latent classes by means of the EM algorithm. Such being the case, first, the partial ranking model was applied to only Data $1,2,3,4$, and 5 ; second, only three sets of data, Data 1, 2, and 3, were analyzed for $T=1,2$, and 3 by all three variants of the latent class model; third, Data $4, \ldots, 9$ were analyzed setting $T=1$ beforehand.

Table 2 summarizes the AIC values given by those analyses. From the table we can observe the following: First, when $n$ is relatively small ( $n \leqq 6)$, the two-stage models fit the data better than the threshold model, whereas the threshold model provides better 
Table 1

Summary of the method of nine any $/ n$ picking experiments

\begin{tabular}{ccllr}
\hline $\begin{array}{c}\text { Data } \\
\text { names }\end{array}$ & $\begin{array}{c}\text { No. of } \\
\text { stimuli }\end{array}$ & \multicolumn{1}{c}{ Stimuli } & Evaluation criteria & $\begin{array}{c}\text { No. of } \\
\text { subjects }\end{array}$ \\
\hline Data 1 & 4 & Soft drinks & Preference & 95 \\
Data 2 & 4 & Four seasons & Preference & 102 \\
Data 3 & 5 & Hot drinks & Preference & 95 \\
Data 4 & 6 & Fruits & Preference & 102 \\
Data 5 & 6 & Japanese verbs & Similarity in meaning & 95 \\
Data 6 & 7 & Purposes for dressing & Importance & 102 \\
Data 7 & 7 & School subjects & Preference & 95 \\
Data 8 & 8 & Districts of the world & Preference & 95 \\
Data 9 & 9 & Alphabets & Loveliness of shape & 95
\end{tabular}

Table 2

Summary of the analyses of nine sets of any $/ n$ picking data by the three variant latent class models

\begin{tabular}{|c|c|c|c|c|c|c|c|}
\hline \multirow{3}{*}{$\begin{array}{c}\text { Data } \\
\text { names }\end{array}$} & \multirow{3}{*}{$T$} & \multicolumn{6}{|c|}{ Response models } \\
\hline & & \multicolumn{2}{|c|}{ Thr. } & \multicolumn{2}{|c|}{ Par. } & \multicolumn{2}{|c|}{ Dic. } \\
\hline & & $d(\alpha)$ & AlC & $d(\alpha)$ & $\mathrm{AIC}$ & $d(\alpha)$ & AIC \\
\hline \multirow[t]{3}{*}{ Data 1} & 1 & 4 & 499.5 & 6 & 491.5 & 6 & 493.9 \\
\hline & 2 & 9 & 486.8 & 10 & $475.2^{\text {*a! }}$ & 10 & $475.2^{*}$ \\
\hline & 3 & 14 & 483.0 & 14 & 483.1 & 14 & 482.8 \\
\hline \multirow[t]{3}{*}{ Data 2} & 1 & 4 & 528.9 & 6 & 525.8 & 6 & $524.6^{*}$ \\
\hline & 2 & 9 & 535.6 & 10 & 532.7 & 10 & 530.0 \\
\hline & 3 & 14 & 539.8 & 14 & $54 \mathrm{I} .1$ & 14 & 536.1 \\
\hline \multirow[t]{4}{*}{ Data 3} & 1 & 5 & 605.6 & 8 & 599.0 & 8 & 595.2 \\
\hline & 2 & 11 & 602.6 & 13 & 596.6 & 13 & 595.1 \\
\hline & & & & & (Dic.-Eq.) & 11 & $592.4 *$ \\
\hline & 3 & 17 & 606.2 & 18 & 604.9 & 18 & 601.2 \\
\hline Data 4 & 1 & 6 & 807.6 & 10 & 789.4 & 10 & $789.3 *$ \\
\hline Data 5 & 1 & 6 & $358.6^{*}$ & 10 & 360.7 & 10 & 361.2 \\
\hline Data 6 & 1 & 7 & $773.3^{*}$ & & & 12 & 782.8 \\
\hline Data 7 & 1 & 7 & $703.4^{*}$ & & & 12 & 714.2 \\
\hline Data 8 & 1 & 8 & $836.8^{*}$ & & & 14 & 839.3 \\
\hline Data 9 & 1 & 9 & $1026.5^{*}$ & & & 16 & 1031.6 \\
\hline
\end{tabular}

accounts for the data when $n \geqq 6$. Second, two-latent class models are appropriate for Data 1 and 3 while a one-class model for Data 2. Among these models the dichotomizing model is most descriptive of all three sets of data.

Notice, in particular, that for Data 3 we tested equality constraints assumed between the preference scale values in different latent classes. In general, equality constriant assumptions help to reduce the number of parameters for distinct preference scale values, and make the resulting scale values more interpretable. For Data 3 we assumed that $a_{11}=a_{12}, a_{21}=a_{22}$, and $a_{51}=a_{52}$ in the dichotomizing variant of the latent class model. This two-class model with the equality constraints, which is referred to as Dic.-Eq. in Table 2, 
Table 3

The estimates of the latent class probabilities and the preference scale values for Data 1 and 3 obtained by the best fitting models

a) The estimated latent class probabilities $\left(\pi_{i}\right)$

\begin{tabular}{ccc}
\hline Data names & Class ] & Class 2 \\
\hline Data 1 & .557 & .443 \\
Data 3 & .421 & .579 \\
\hline
\end{tabular}

b) The estimated preference scale values $\left(a_{i}\right)$, setting $s_{t}=1$

\begin{tabular}{llrr}
\hline Data names & Stimuli & Class I & Class 2 \\
\hline Data 1 & Isotonic drink & 4.82 & .32 \\
& Orange juice & -.60 & 1.72 \\
& Cola & -.03 & .96 \\
& Soda-pop & -.91 & 1.00 \\
& & & \\
Data 3 & Cocoa & .66 & .66 \\
& Green tea & .49 & .49 \\
& Coffee & 1.35 & -.55 \\
& Milk & -.94 & .14 \\
& Tea & 1.02 & 1.02
\end{tabular}

Table 4

The conditional probabilities $z[M(r)]$ that each any/n picking response $M(r)$ originated from each latent class $t$ for Data 3

\begin{tabular}{|c|c|c|c|c|}
\hline \multirow{2}{*}{$r$} & \multirow{2}{*}{ Response patterns $M(r)$} & \multirow{2}{*}{ Frequencies } & \multicolumn{2}{|c|}{ Probabilities $w_{i}[M(r)]$} \\
\hline & & & Class 1 & Class 2 \\
\hline 1 & {$[\mathrm{M}]^{\mathrm{a})}$} & 3 & .075 & .925 \\
\hline 2 & [GT] & 5 & .180 & .820 \\
\hline 3 & [G] & 2 & .367 & .633 \\
\hline 4 & [GC] & 2 & .947 & .053 \\
\hline 5 & {$[\mathrm{CT}]$} & 11 & .934 & .066 \\
\hline 6 & {$[\mathrm{GCT}]$} & 4 & .927 & .073 \\
\hline 7 & {$[\mathrm{MT}]$} & 2 & .030 & .970 \\
\hline 8 & [O] & 5 & .367 & .633 \\
\hline 9 & [OGMT] & 5 & .000 & 1.000 \\
\hline 10 & {$[\mathrm{C}]$} & 2 & .948 & .052 \\
\hline 11 & [TO $]$ & 11 & .175 & .825 \\
\hline 12 & [CMT] & 1 & .630 & .370 \\
\hline 13 & [OGM] & $i$ & .012 & .988 \\
\hline 14 & [TGO] & 9 & .038 & .962 \\
\hline 15 & [GTM] & 2 & .007 & .993 \\
\hline 16 & [OGCMT] & 6 & .500 & .500 \\
\hline 17 & {$[\mathrm{OCT}]$} & 4 & .918 & .082 \\
\hline 18 & [OMT] & 2 & .006 & .994 \\
\hline 19 & [OGCT] & 6 & .885 & .115 \\
\hline 20 & [OC] & 5 & .945 & .055 \\
\hline 21 & {$[\mathrm{~T}]$} & 4 & .367 & .633 \\
\hline 22 & {$[\mathrm{OG}]$} & 2 & .209 & .791 \\
\hline 23 & {$[\mathrm{GM}]$} & 1 & .037 & .963 \\
\hline
\end{tabular}

a) "O", "G", "C", "M", and "T" stand for cocoa, green tea, coffee, milk, and tea, respectively. 
showed a better fit than the corresponding model without equality constraint assumptions.

Furthermore, it may be worthwhile and interesting to examine the results of the analyses of Data 1 and 3 in more detail. Table 3 contains the latent class probabilities and the preference scale values obtained by the best fitting models, i.e., the dichotomizing model with $T=2$ latent classes for Data 1, and the dichotomizing model with $T=2$ latent classes assuming the equality constraints for Data 3.

We can see from the table that for Data 1 the differences in the corresponding scale values between classes 1 and 2 imply that class 1 likes isotonic drink (e.g., brands such as Pocarisweat and Aquarius) much better than the remaining soft drinks. In contrast, class 2 favors orange juice and soda-pop over isotonic drink. These results indicate that isotonic drink serves as a key to the classification of subjects into two groups: the isotonic drink group (class 1) and the juice-soda group (class 2).

Next, look at the estimates of the preference scale values for Data 3. Owing to the equality constraints, we can easily observe that the two latent classes definitely differ with respect to the preference scale values of coffee and milk. That is, subjects belonging to class 1, the coffee group, prefer coffee above all the other hot drinks and prefer milk least of all. In contrast, subjects belonging to class 2, the anti-coffee group, like coffee least of all. To sum up, we can infer that subjects are classified into two groups according to whether or not they like coffee. In addition, see Table 4, which presents the conditional probabilities $2 \%[M(r)]$ that response pattern $M(r)$ originated from latent class $t$. These probabilities demonstrate that response patterns containing coffee occur almost exclusively to subjects belonging to class 1 . In contrast, those responses choosing milk but rejecting coffee come from subjects in class 2 with probability $w_{2}[M(r)] \geqq .925$.

\section{Discussion}

1. The partial ranking and the dichotomizing models have one weakness in common. That is, no substantial assumptions are made about the probabilities $u_{m}$. Most likely, these probabilities are related in some way or other to the character of subjects participating in the experiment (e.g., they may be apt to choose a lot of stimuli as most preferred), to the nature of stimuli (e.g., only a few of them attract subjects), to the number of stimuli used as alternatives, and so forth. It is desirable, therefore, to find what variables are truly relevant in subjects' choosing the NSC numbers, and to clarify how those variables are related to $u_{m}$. This will be the subject for future study.

2. As was noted earlier, the number of all possible any $/ n$ picking patterns is $2^{n}-1$. Hence, it often occurs that many of the expected frequencies are too small to justify the application of the EM algorithm. Although no satisfactory solutions in these cases are presently known, the strategy of grouping response patterns until their expected frequencies exceed a specified minimum value (Bock \& Aitkin, 1981) has proven effective for assessing the fit of a model and determining the number of latent classes. An undesirable feature of this procedure is that patterns may be grouped in different ways, and each particular grouping may not lead to the same conclusion (Böckenholt \& Böckenholt, 1991). One practical way to overcome this limitation on the applicability of the present procedure is to impose a restriction on the range of the number of stimuli the subject is allowed to choose as most preferred, or to prescribe that number prior to the any/n picking experiment. These treatments will drastically reduce the number of possible any $/ n$ picking response patterns to be produced by subjects. With minor modification the latent class formulations given in this paper can be applied to such data as well.

3. In the present studies we imposed no 
structures on the preference scale values such as those in an ideal-point model or a preference vector model mainely because our major purpose was to compare the three different response models of any $/ n$ picking behavior, and partly because pilot studies suggested that when $n$ is small, say $n \leqq 7$, and when the number of latent classes assumed is rather small, the unrestricted model is a better model for the representation of preferences than the ideal-point model.

\section{Concluding Remarks}

In the previous latent class analyses of any/ $n$ picking data only the threshold model was implemented as the response model excluding other possibilities. The major advantage of the present latent class procedure over the previous ones is that the former incorporates three plausible response models of any $/ n$ picking behavior in the latent class models so that we can compare their goodness of fit and choose the one which better fits a particluar data set. The present analyses have revealed that the two-stage response models developed in this paper provide better accounts of the data for $n \leqq 6$ while the conventional threshold model is more descriptive of the data for $n \geqq 6$. We can infer from this result that subjects perform an any/ $n$ picking task in different manners according to the number of alternative stimuli. We are planning on extending the present procedure to the analysis of sorting data.

\section{References}

Akaike, H. 1974 A new look at the statistical model identification. IEEE Transactions on Automatic Control, 19, 716-723.

Bock, R.D., \& Aitkin, M. 1981 Marginal maximum likelihood estimation of item parameters: Application of an EM algorithm. Psychometrika, 46, 443-459.

Böckenholt, U., \& Böckenholt, I. 1991 Constrained latent class analysis: Simultaneous classification and scaling of discrete choice data. Psychometrika, 56, 699-716.

Croon, M.A. 1989 Latent class models for the analysis of rankings. In G. De Soete, H. Feger \& K.C. Klauer (Eds.), New developments in psychological choice modeling. Amsterdam: North-Holland. Pp. 99-121.

Goodman, L.A. 1979 On the estimation of parameters in latent structure analysis. Psychometrika, 44, 123-128.

Hojo, H. 1986 Response threshold models for binary ranking and sorting. Behaviormetrika, 20, 1-12.

Lazarsfeld, P.F., \& Henry, N.W. 1968 Latent structure analysis. New York: Houghton Mifflin.

Luce, R.D. 1959 Indinidual choice behavior: $A$ theoretical analysis. New York: Wiley.

Takane, Y. 1981 Multidimensional successive categories scaling: A maximum likelihood method. Psychometrika, 46, 9-28.

Takane, Y. 1983 Choice model analysis of the "pick any/ $\mathrm{n}$ " type of binary data. Handout at the European Psychometric and Classification Meetings, Jouy-en-Josas, France.

Takane, Y., \& Carroll, J.D. 1981 Nonmetric maximum likelihood multidimensional scaling from directional rankings of similarities. Psychometrika, 46, 389-405.

Takane Y., \& de Leeuw, J. 1987 On the relationship between item response theory and factor analysis of discretized variables. Psychometrika, 52, 393-408.

Tone, K. 1981 BASIC. Tokyo: Baifukan. (In Japanese)

(Received June 24, 1992; accepted Nov. 14, 1992) 